\title{
Multiprotocol Label Switching in Vehicular Ad hoc Network for QoS
}

\author{
*Kashif Naseer Qureshi, Abdul Hanan Abdullah \\ Universiti Teknologi Malaysia, Malaysia \\ *kashifnq@gmail.com
}

\begin{abstract}
Vehicular Ad hoc Networks (VANET) provides a wireless communication between vehicles. VANET applications play a significant role in the transportation sector such as vehicle safety, environmental efficiency, traffic control etc. Vehicular Ad hoc network is a subclass of Mobile Ad hoc networks. One of the main concerns in transportation is quality of service (QoS). In VANET, various solutions proposed for quality of services and these solutions applied on layer 2 and layer 3. In this paper, we proposed a Multiprotocol Label Switching. MPLS is an efficient and effective technique that forwards the packets across the network by using the contents of the labels attached to the IP packets. MPLS is known to be a layer 2.5 technology because it supports both data link layer or layer- 2 and layer-3. The use of MPLS as backbone networks has increased over the past few years as compared to traditional IP networks, which were based on Iayer-2 technologies. MPLS is a forwarding method used for backbone network. In this paper, we improve quality of service in term of delay, packet loss and throughput in highway areas.
\end{abstract}

Keywords: Vehicular Ad hoc Network VANET, Multiprotocol Label Switching MPLS, Quality of service (QoS)

\section{Introduction}

The quality in services are describes as a service for successful packet delivery or data sending between source to destination without any error. The wireless network has some metrics to deliver the packet between two nodes such as high bandwidth, reliability, through put, and other quality of service (QoS). Many applications need high quality for delivering the services such as video conferencing (VC) and voice over IP (VoIP), (Gospodinov, 2004),multimedia services, video Security. The quality of service is a main requirement for these application and services. Researchers have done considerable work for examine and enhance QoS performance in wireless and wired communication. Quality of services protocols have been applied for reducing delay, latency, and jitter problems such as Intserv (Integrated services), Diffserv (Differentiated services) and Multiprotocol label switching (MPLS). The MPLS technology allows a certain degree of QoS in packet forwarding. In this paper, we discuss the MPLS as a backbone in VANET for increasing the quality of service (QoS). The paper is structured as follows: Section two elaborates a concise impression of Multiprotocol label switching and in third section explanation of vehicular Ad hoc network and their architecture.

Multiprotocol label switching: Many IT companies are looking for an appropriate solution for their wide area networks. They purchased frame relay or ATM leased lines in the recent past. Virtual private network provides maximum security and a tunneled route for data traffic over the Internet and these technologies work on layer-2 data link layer. These technologies make secure and protected from intruders and hackers that pose a threat to the backbone networks. VPNs based networks faced the scalability problem (Ooms, et al., 2002). To overcome this problem MPLS was introduced (Daugherty \& Metz, 2005), MPLS is an efficient and effective technique that forwards the packets across the network by using the contents of the labels attached to the IP packets (Alwayn, 2001). MPLS is known to be a layer 2.5 technology because it supports both data link layer and network layer. Multiprotocol Label Switching (MPLS) is similar to the virtual circuit concept used in technologies such as ATM. For finding the next hop in network the routing table is a time consuming job but through Multiprotocol Label Switching (MPLS), routers advance packets by seem to be the label of a packet. The labels, which are attached with packet because the network layer functions such as routing and forwarding execute independently from data link layer functions like switching. The MPLS runs frame relay, Ethernet and ATM that are layer 2 technologies. The use of layer 2 technologies is one of the most noticeable points. Traffic engineering is another considerable character of MPLS. The two most implemented protocols Open Shortest Path First (OSPF) (Moy, 1998) and Routing Information Protocol (RIP) (Hedrick, 1988) are typically based on protocol design and metrics for finding the shortest path and miss some other important metrics like packet loss, delay, jitter, throughput and congestion are not considering. In case of MPLS, the 
determination on the most excellent route "which organize service requirement of packets and the shortest path" is not necessarily for this.

\section{Figure 1: Multiprotocol label switching (MPLS) Domain Architecture}

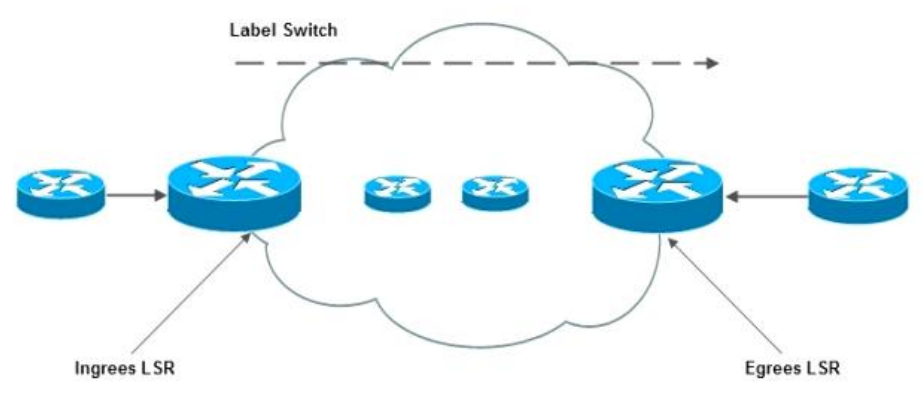

Figure 2: Vehicle Network

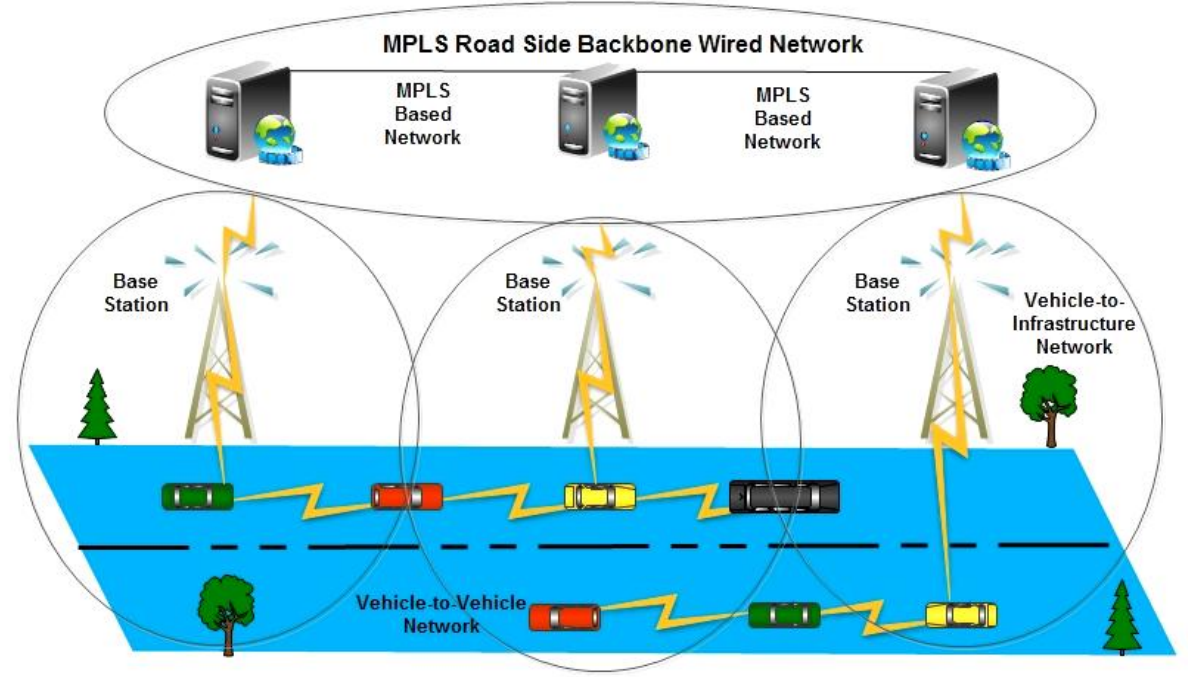

\section{Vehicular Ad hoc Networks}

In Ad hoc Networks, the mobile nodes are using multi-hop wireless links for communication and when this Ad-hoc technology implements in the transportation sector, so it is called VANET. The Vehicular AdHoc networks are self-configuring and make available communications necessary to organize Intelligent Transportation Systems (ITS). The VANET provides the facility to ensure wireless network between vehicles nodes on the urban and highway environments. Vehicular Ad-Hoc network (VANET) is a unique type of Mobile ad-Hoc networks (MANET) but with own unique characteristics. In VANET the nodes are operational with on- board computers, sensors, and element of roadside infrastructure and pedestrian personal devices smart phones etc. The vehicular Ad hoc networks provides various types of applications services for public-safety and have a wide range of infotainment applications for example audio applications, video applications, e-map, internet services. The two types of communication present in VANET the first one is communication between vehicles and other one is communication between vehicles and roadside unit. The VANET architecture based on the roadside unit (RSU) for vehicle-toinfrastructure communication. The roadside unit (RSU) needs a backbone network for communication with other roadside units and for internet services (Kiani \& Baig, 2010). Roadside backbone network (RBN) and V2V, V2I shown in below figure. All application of vehicular Ad hoc networks have own quality of service (QoS), and they are divided into two main categories safety applications and infotainment applications. In the first category, the applications like warning and accident detection and in these applications packets need minimum End-to-End delay and high priority because if the packets are not in time so the result of that is life loss or other accidents. A second type of applications provides entertainment services to the user or travelers. When we using more efficient MPLS service for backbone 
network so the quality of service, (QoS) will improve (Fathy, GholamalitabarFirouzjaee, \& Raahemifar, 2012; Vijayarangam \& Ganesan, 2002).

\section{Types of Vehicular Ad hoc Networks}

In abroad scenes, there are two types of networks in vehicular network architecture: wired and wireless depends on the environment. In wired network, the information is carrying through cables such as servers attached with roadside units or the main station. While in the vehicle-to-vehicle network, the wireless data is sent from one vehicle node to another vehicle node through wireless links, this is pure Ad hoc networks. The second type of network is belonging to vehicles to infrastructure. In the vehicle-toinfrastructure (V2I), network roadside unit or base station or access point to connect with wireless vehicle nodes for communication.

Mobility Models: Vehicular Ad hoc network mobility models are categorized into two main categories microscopic and macroscopic. In macroscopic, the motion constrained like roads, street, crossroads and traffic lights, traffic are considered. Distribution of vehicles and generation of vehicular traffic density, flow also defined in this group. The second group is microscopic and in this group movement of each vehicle node and vehicle behavior considered. Different mobility model proposed for emulation of vehicle node in the network, and they are depending on the situation. Below table shows mobility models and their usage.

Table 1: Different types of mobility models with usage

\begin{tabular}{lll}
\hline S/No & Mobility Models & Usage \\
\hline 1 & Reference Point Group Mobility Model (RPGM) & For military battlefield communication \\
2 & Random Waypoint Model (RWM) & For research Community \\
3 & Freeway Mobility Model (FWM) & For freeway motion behavior \\
4 & Manhattan Mobility Model (MHM) & For vehicle node movement \\
\hline
\end{tabular}

For getting the results through simulation, we use Manhattan Mobility Model (MHM) for mobility movement pattern. Usually in this model streets defined by maps and use for urban areas. Many protocols have been proposed for VANET such as topology routing protocol, which are further divided into three types: proactive, reactive and hybrid. DSDV is a most general proactive protocol based on the shortest path (Luo, Gu, Zhao, \& Yan, 2010). These types of protocols have overhead problem because each node maintain information of all connected node in the routing table. In on demand or reactive protocols, routes are discovered and maintained on demand such as Ad hoc on demand reactive routing protocol (AODV)(Shastri, Dadhich, \& Poonia, 2011). AODV solved several problems of proactive protocols by supporting a large number of networks and reducing flooding in the network. Using MPLS in vehicular Ad hoc network as a backbone service for enhancing the network efficiency and performance. Vehicle to infrastructure network where vehicle nodes are connected with the base station and that base station have own service of domain. The base station is connected with a wired network. MPLS improves overall quality of network. We also use AODV because it is suitable for VANET scenario.

MPLS in VANET: MPLS technology introduced in late 90's for quality of service and design for high-speed optical backbone. MPLS is an efficient and effective technique that forwards the packets across the network by using the contents of the labels attached to the IP packets. The use of MPLS as backbone networks has increased over the past few years as compared to traditional IP networks which were based on Iayer-2 technologies i.e. ATM and frame relay. Adopting MPLS in VANET, the Quality of Services (QoS) parameters are efficient such as packet loss, round trip delays, fault tolerant paths, and structural management services. Due to high mobility of vehicles, utilizing MPLS in vehicle nodes may not have a positive effect, so we implement this technology in base stations (BS), and base station is connected with a wired network. The vehicles forward their data through the base station for analyzing and processing to the wired infrastructure. After usage of MPLS, the QoS metrics will be more efficient and gain higher results.

\section{Simulation Results}

We use NS2 simulation which is event driven based simulation and SUMO use for mobility model. Our data set is Manhattan mobility model in this model the vehicles are move along the grid of vertical and horizontal streets. We have created an urban area and with many roads and traffic lights and 
intersections. In our scenario, we use 4 groups of vehicles and each group has 4 vehicles. The simulation parameters are below in table.

Table 2: Simulation Parameters

\begin{tabular}{ll}
\hline Network Area & $\mathbf{5 0 0} * \mathbf{6 0 0} \mathbf{~ M}$ \\
\hline Radio Range & $150 \mathrm{~m}$ \\
Channel & Wireless \\
Traffic type & CBR \\
Routing Protocol & AODV \\
Number of Vehicles & 16 \\
Base stations & 10 \\
Speed of vehicles node & $30 \mathrm{~km} / \mathrm{h}$ \\
Packet size & $800 \mathrm{byte}$ \\
Transport protocol & UDP \\
\hline
\end{tabular}

The proposed design of MPLS is defining as the base stations nodes are MPLS based and connected with wire. In vehicular Ad hoc network, the MPLS implementation on base stations nodes, which are connected through a wire. Through base stations, communication is possible between vehicles and the wired MPLS based domain. In this scenario, each base station has its own domain for addressing in 3 levels of domain. In VANET, the vehicles node are communicating with each other, and base stations send these packets through wired network to the other base station. We improving this communication and getting efficient QoS, we proposed MPLS based domains in wired side. MPLS mechanism is fast and reliable compare to previous mechanisms. Through this technique, we attain improved QoS results in term of end-to-end delay, packet loss and throughput (Nzouonta, Rajgure, Wang, \& Borcea, 2009).MPLS provides faster forwarding compare to IP nodes.

\section{Fig 3: Vehicle-to-Vehicle and Vehicle -to Infrastructure Networks in VANET}

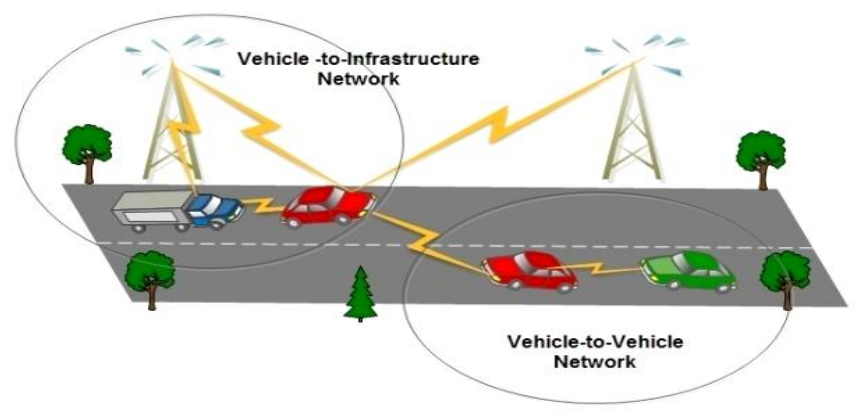

We compare V2V with AODV based communication with Vehicle-to-infrastructure MPLS based communication. The results show the better performance of vehicle -to-infrastructure network. Graphs show the overall performance of V2V routing and V2I MPLS enabled routing.

Figure 4: Throughput

\section{Throughput}

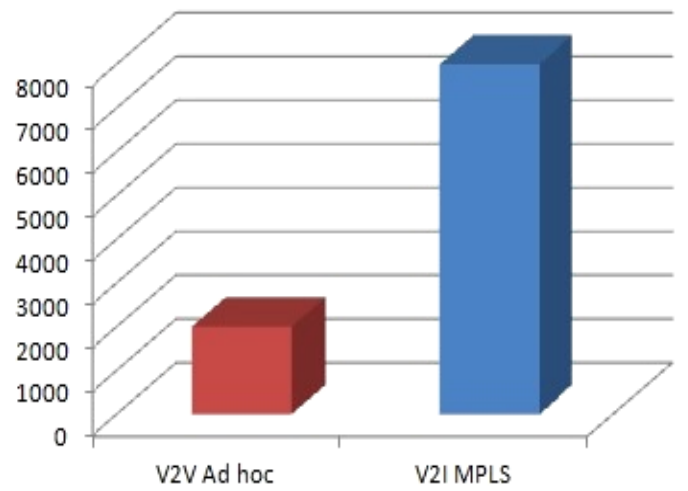


Packet Loss

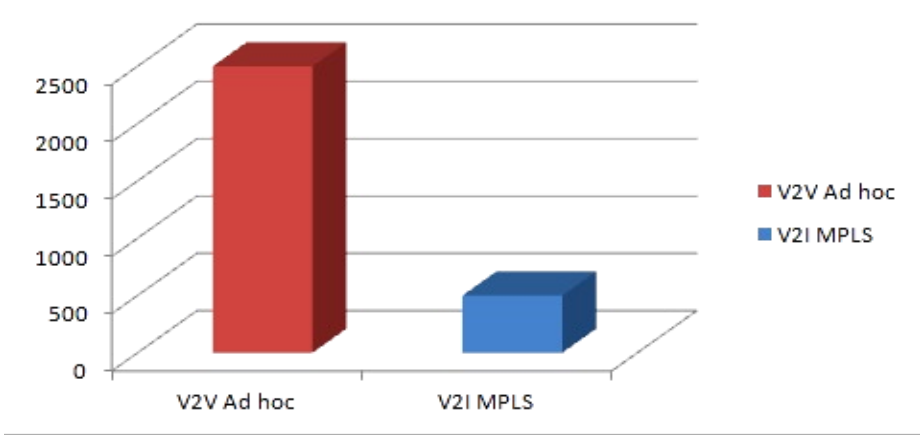

Figure 6: End-to-End Delay

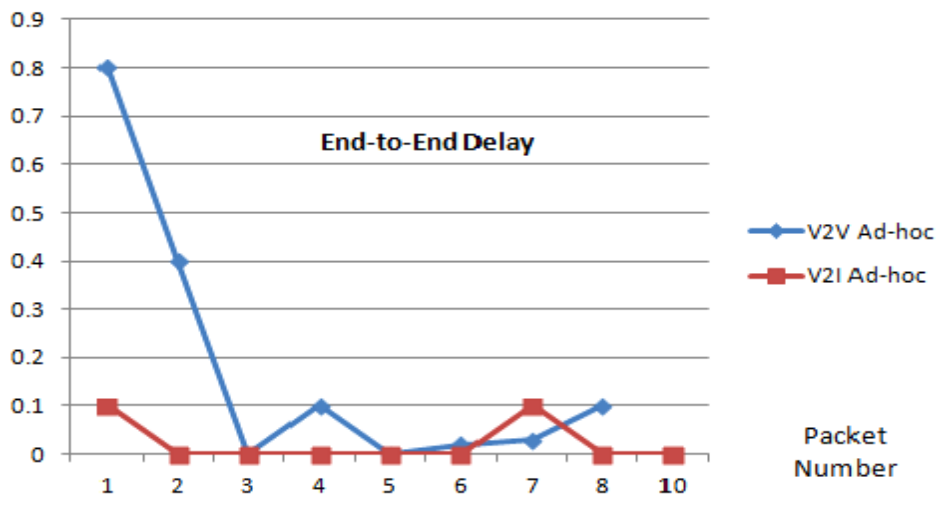

The above graphs show the AODV protocol performance in Vehicle-to-vehicle network. Lots of packets drop and performance is poor, and when we check the performance of vehicle-to-infrastructure MPLS based network, so performance is better than previous. Using of MPLS in the backbone network we overcome the packet loss problem in AODV Ad hoc routing.

\section{Conclusion}

In Vehicular network, the mobility is very high, and packets loss problem still get the intension of researchers. In this paper, the usage of MPLS in VANET in urban city areas is an efficient technique to overcome the packet loss end-to-end delay and enhance throughput for improving the QoS presented. Vehicles are connecting with each other and with base stations and base stations are typically connected with wireless, but we proposed the base stations are connected with MPLS mechanism and they are wired based network. Through simulation, we proved that this method is better and efficient in some QoS parameters like throughput, end-to-end delay, packet loss.

\section{References}

Alwayn, V. (2001). Advanced MPLS design and implementation: Cisco Press.

Daugherty, B. \& Metz, C. (2005). Multiprotocol label switching and IP. Part I. MPLS VPNs over IP tunnels. Internet Computing, IEEE, 9(3), 68-72.

Fathy, M., GholamalitabarFirouzjaee, S. \& Raahemifar, K. (2012). Improving QoS in VANET Using MPLS. Procedia Computer Science, 10, 1018-1025.

Gospodinov, M. (2004). The affects of different queuing disciplines over FTP, Video and VoIP Performance. Paper presented at the International Conference on Computer Systems and Technologies-CompSysTech '2004.

Hedrick, C. L. (1988). Routing information protocol.

Kiani, H. S. \& Baig, M. H. (2010). Performance Evaluation of MANET Using MPLS. MS thesis, Bleking Institute of Technology, Sweden. 
Luo, J., Gu, X., Zhao, T. \& Yan, W. (2010). A mobile infrastructure based VANET routing protocol in the urban environment. Paper presented at the Communications and Mobile Computing (CMC), 2010 International Conference on.

Moy, J. (1998). Open shortest path first version 2: STD 54, RFC 2328, April.

Nzouonta, J., Rajgure, N., Wang, G. \& Borcea, C. (2009). VANET routing on city roads using real-time vehicular traffic information. Vehicular Technology, IEEE Transactions on, 58(7), 3609-3626.

Ooms, D., Sales, B., Livens, W., Acharya, A., Griffoul, F. \& Ansari, F. (2002). Overview of IP multicast in a multi-protocol label switching (MPLS) environment. IETF RFC3353.

Shastri, A., Dadhich, R. \& Poonia, R. C. (2011). Performance Analysis Of On-Demand Routing Protocols For Vehicular Ad-Hoc Networks. International Journal of Wireless \& Mobile Networks (IJWMN), 3, 103111.

Vijayarangam, S. \& Ganesan, S. (2002). QoS implementation for MPLS based wireless networks. Paper presented at the ASEE Conference. 